\title{
Insulin adherence and the associated factors among patients with type 2 diabetes mellitus at the Hospital Queen Elizabeth II, Sabah
}

\author{
Siew Yee Yong ${ }^{1}$ (D) $\cdot$ Greta Miranda Goh ${ }^{2} \cdot$ Huai Heng Loh $^{2}$ \\ Received: 8 July 2020 / Accepted: 9 November 2020 \\ (C) Springer-Verlag GmbH Germany, part of Springer Nature 2020
}

\begin{abstract}
Aim The purposes of this study were to: (a) determine the insulin adherence rate and reasons for non-adherence among patients with T2DM, (b) determine the relationship between the Health Belief Model (HBM) construct and insulin adherence, (c) assess the patient's characteristics that influence the Health Belief Model (HBM) construct, and (d) identify the predictors of insulin non-adherence among patients with T2DM.

Subject and methods An analytical cross-sectional survey was carried out on 360 T2DM outpatients at the Diabetes Clinic in Hospital Queen Elizabeth II (HQE II), Sabah. Insulin adherence was measured using a validated Medication Compliance Questionnaire (MCQ). Results A total of 360 patients took part in this study. Majority were female (60.6\%) with an age range from 28 to 87 years (54.07 \pm 13.62 years). The overall prevalence of insulin adherence was $60.3 \%$. Forgetfulness was the most common factor for insulin non-adherence $(66.9 \%)$. The majority of the respondents showed positive perceptions and beliefs of their insulin treatments, with a mean $71.11(\mathrm{SD}=9.59)$. There was a significant correlation between insulin adherence and all the HBM constructs. Overall, the HBM constructs predicted $40.9 \%$ of the variance in insulin adherence. The strongest predictor of insulin adherence was perceived benefits followed by self-efficacy.

Conclusions The current study suggests an acceptable level of insulin adherence among the T2DM outpatients in HQE II. It is recommended that the high-risk group of non-adherence should be identified, the insulin regimen adjusted, and an individual intensive health education designed in order to increase insulin adherence.
\end{abstract}

Keywords Type 2 diabetes mellitus $\cdot$ Insulin adherence $\cdot$ Barriers $\cdot$ Perceptions and beliefs $\cdot$ Depression

\section{Introduction}

T2DM is a progressive chronic disease due to the pancreas failing to produce enough insulin, or the body cells being

Siew Yee Yong

yongsiewyee81@gmail.com

Greta Miranda Goh

kcgoh@unimas.my

Huai Heng Loh

hhloh@unimas.my

1 Malaysian Ministry of Health Training Institution Kota Kinabalu, Jalan Kolam, Bukit Padang, 88400 Kota Kinabalu, Sabah, Malaysia

2 Faculty of Medicine and Health Science, Universiti Malaysia Sarawak (UNIMAS), Jalan Datuk Mohammad Musa, 94300 Kota Samarahan, Sarawak, Malaysia unable to utilize the insulin produced by the pancreas effectively, resulting in hyperglycaemia (ADA 2012). Treatment for diabetes includes lifelong self-care practices in controlling and managing diabetes. These self-care practices include medication adherence, exercise habits, dietary modification, and self-blood glucose monitoring. However, not all diabetic patients perform their self-care activities as recommended (Gunggu et al. 2016). Among these self-care activities, adherence to insulin treatment is of the utmost importance, as patients on insulin treatments have either inadequate glycemic control or newly diagnosed T2DM with $\mathrm{HbAlc}$ more than $10 \%$ (MOH 2015). Insulin is a strong medication for lowering blood glucose levels and reduces the risk of developing diabetic complications. If diabetic patients do not adhere well to their insulin treatment, the progression of the disease is elevated and leads to early morbidity, poor quality of life, and the imposition of a significant burden on both the patient and the country. 